\title{
Crabronidae and Sphecidae (Hymenoptera: Apoidea) type specimens deposited in the Museu de Zoologia da Universidade de São Paulo, Brazil
}

\author{
Juliana Menezes de Jesus ${ }^{1,3}$; Helena Carolina Onody ${ }^{2}$; Kelli dos Santos Ramos ${ }^{1,4}$; Tamires de Oliveira Andrade ${ }^{1,5}$; \\ Alvaro Doria dos Santos ${ }^{1,6}$ \& Carlos Roberto Ferreira Brandão ${ }^{1,7}$
}

\footnotetext{
1 Universidade de São Paulo (USP), Museu de Zoologia (MZUSP). São Paulo, SP, Brasil.

${ }^{2}$ Universidade Estadual do Piauí (UESPI), Laboratório de Zoologia. Corrente, PI, Brasil. ORCID: http://orcid.org/0000-0003-3570-8183. E-mail: helenaonody@gmail.com

${ }^{3}$ ORCID: http://orcid.org/0000-0003-1312-2018. E-mail: julinaaabio60@gmail.com

${ }^{4}$ ORCID: http://orcid.org/0000-0003-1453-5885.E-mail: kellisramos@gmail.com

${ }^{5}$ ORCID: http://orcid.org/0000-0001-9492-1967.E-mail: tamiresandrade@usp.br

${ }^{6}$ ORCID: http://orcid.org/0000-0002-7997-4195. E-mail: alvarods3@gmail.com

7 ORCID: http://orcid.org/0000-0002-4689-5845. E-mail: crfbrand@usp.br
}

\begin{abstract}
This catalogue lists the type specimens of Crabronidae and Sphecidae (Hymenoptera: Apoidea) deposited in the Museu de Zoologia da Universidade de São Paulo, Brazil (MZUSP). The collection includes a total of 83 type specimens (17 holotypes, 66 paratypes), 82 of which belong to nine genera and 35 species of (rabronidae and only one of Sphecidae. All labels contents and additional information obtained from other available sources are presented. High resolution photographs of the primary types are also provided.
\end{abstract}

Key-Words. Insecta; MZUSP; Neotropical; Taxonomy; Wasp.

\section{INTRODUCTION}

The Hymenoptera collection of the Museu de Zoologia da Universidade de São Paulo (MZUSP) is one of the world's most expressive considering the Neotropical fauna of Hymenoptera, preserved both in dry (pinned) and wet (alcohol) conditions. First specimens' records date back to early twentieth century, when the museum at the time was still the Department of Zoology of the Museu Paulista (Klingenberg \& Brandão, 2005). Since the specimens collected by Hermann von Ihering (18501930) and by Hermann von Luderwadt (18651934), the collection has grown notably through various collecting projects efforts, acquisition of other collections, exchange with colleagues and institutions, and through donations of specimens from researchers of all over the world.

In the last few years, the Brazilian agencies CNPq (Conselho Nacional de Desenvolvimento Científico e Tecnológico) and FAPESP (Fundação de Amparo à Pesquisa do Estado de São Paulo) financed the development of several projects regarding the Hymenoptera collection of the
MZUSP, to improve its curatorial capacity and to make information available to researchers and other society segments. In this context, and taking into account the recommendation $72 \mathrm{~F}$ of the International Code of Zoological Nomenclature (ICZN, fourth edition, January 1999) about the role of collections in making the types available to researches, catalogues have been published aiming to list all Hymenoptera types specimens deposited in the MZUSP collection. These catalogues cover, until this moment: ants (Brandão, 1991; Klingenberg \& Brandão, 2005; Brandão et al., 2010; Esteves et al., 2011; Prado \& Brandão, 2013; Ulysséa \& Brandão, 2013; Ulysséa et al., 2015), bees (Ramos et al., 2015), "Symphyta" \& "Parasitica” wasps (Onody et al., 2014), Chrysidoidea (Santos et al., 2017) and Pompiloidea, Thynnoidea and Vespoidea wasps (Andrade et al., 2018). The most recent ones include also images of the primary types to avoid long distance loans and unnecessary movement of these specimens.

The aim of this paper is to catalog the type specimens of the Crabronidae and Sphecidae (Hymenoptera: Apoidea) deposited in the MZUSP. 


\section{MATERIAL AND METHODS}

This catalogue lists all types of Crabronidae and Sphecidae specimens deposited in the Museu de Zoologia da Universidade de São Paulo (MZUSP). There are no type specimens of other Apoidea families in the collection, except bees already cataloged by Ramos et al. (2015). Species data are organized by families, subfamilies, tribes, genera and species in alphabetical order according to the current classification and their original combinations. We adopted the subfamilies classification of Crabronidae as in Amarante (2006). Type specimen's current status were verified by the following databases:Taxonomic Catalog of the Brazilian Fauna (Oliveira et al., 2018), Integrated Taxonomic Information System (ITIS), Hymenoptera Online (HOL) and Catalog of Sphecidae of the Academy of Sciences of California (Pulawski, 2018). The names of species are followed by author(s), year, and page of publication.

High resolution photographs of the primary types were obtained with a Leica DFC 295 video camera attached to a Leica M205C magnifying stereoscope. Leica LAS (Leica Application Suite V3.6.0) and Helicon Focus (version 6.0.18) software were used to assemble the series of images taken at different focus distances by z-stacking method.

All information given herein was compiled from label data, MZUSP records, published material and other available sources that we were able to recover. Whenever possible, data are presented in the following sequence: type category, number and sex of specimens, current MZUSP registration number, locality (country, state/province, city, and other locality details), date of collecting, trap type or other collecting technique and collector's name. Labels information were transcribed between apostrophes (' '), sometimes indicating information from different labels attached to the same specimen. Abbreviated and additional information are presented in brackets ([ ]). Original data are provided in the original languages of the labels and additional information in English.

\section{RESULTS}

The collection comprises a total of 83 type specimens, 82 of them belong to Crabronidae (17 holotypes and 66 paratypes of nine genera and 35 species) and one holotype is a Sphecidae.

\section{Apoidea \\ Crabronidae \\ Bembicinae \\ Bembicini \\ Liogorytes Bohart}

\section{Liogorytes brasilicus Bohart, 2000: 207 (Fig. 1A-D)}

Holotype: \& (\#MZSP 61966) '[Brazil], São Paulo,

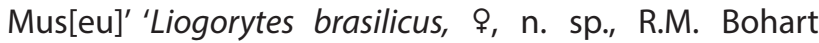
det.' 'Holotype, 'Liogorytes brasilicus, ९, R.M. Bohart'.

\section{Stictia lliger}

\section{Stictia maculitarsis Schrottky, 1913: 705 (Figs. 2A-D)}

Holotype: ᄋ (\#MZSP 61957) '[Brazil], E.[stado] S.[ão] Paulo, Franca, 15389 [Schrottky col., 1903]' 'Stictia o maculitarsis, type! n. sp., C. Schrottky det. 1910' 'Holotypus'.

Comments: Rasmussen et al. (2009) did not locate the type of Sticta maculitarsis. However, the listed specimen bears labels that agree with the original description made by Schrottky.

\section{Crabroninae \\ Bothynostethini \\ Bohartella Menke}

\section{Bohartella hypopsia Menke \& Vardy, 1980: 82}

Paratype: 1 ơ (\#MZSP 61958) 'Brasil, [São Paulo], Campinas, Mar.[ço], 1924' 'F.X. Williams collector' '\#90 Scapheutes o' 'Hyposia Menke + Vardy'.

\section{Crabronini \\ Podagritus Spinola}

Podagritus jordaonis Leclercq, 2000: 37 (Fig. 3A-D)

Holotype: 9 (\#MZSP 61959) '[Brazil], São Paulo, Campos do Jordão, 23.XII.1944, F. Lane col.' 'J Leclercq dt, Podagritus jordaonis Leclercq n. sp. O' 'Holotype'.

\section{Podagritus ypirangae Leclercq, 2000: 50 (Fig. 4A-D)}

Holotype: 9 (\#MZSP 61960) ‘[Brazil], Ypiranga [lpiranga], E.[stado] S.[ão] Paulo, i.[19]96' 'J Leclercq dt, Podagritus ypirangae Leclercq ơ' 'Holotype' ‘97839'.

\section{Larrini Tachysphex Kohl}

Tachysphex advenus Pulawski, 1974: 32 (Fig. 5A-D)

Holotype: ơ (\#MZSP 61961) '[Brazil], S.[ão] P.[aulo], Boa Esperança do Sul, Faz.[enda] Itaquerê, 21.VII.1964 K. Lenko col.' 'KL.47' 'HOLOTYPUS, Tachysphex advenus, o', W.J. Pulawski, 1973'.

Paratypes: $10^{\top}$ and 19 (\#MZSP 61962, 61963) '[Brazil], S.[ão] P.[aulo], Boa Esperança do Sul, Faz.[enda] Itaquerê, 22.vi.1965, K. Lenko col.' 'Paratypus, Tachysphex advenus, W.J. Pulawski, 1973'.

Comments: The original publication reports 3 paratypes deposited in the MZUSP, however, one male was not located. 


\section{Tachytes Panzer}

\section{Tachytes catarinae Bohart, 1978: 452}

Paratype: 1 ơ (\#MZSP 61964) '[Brazil], São Paulo, Onda Verde, Faz.[enda] São João, Jan.[eiro], 1946, F. Lane col.' 'Paratype, Tachytes catarinae, o', R.M. Bohart [col.]'.

\section{Tachytes coloratus Bohart, 1978: 457}

Paratype: 1 \& (\#MZSP 61965) '[Brazil], São Paulo, Nova Europa, Faz.[enda] Itaquerê, 28.xi.1963, K. Lenko col.' 'Paratype, Tachytes coloratus, ९, R.M. Bohart'.

\section{Tachytes fraternoides Bohart, 1978: 462}

Paratypes: 1 o' (\#MZSP 62276) '[Brazil], Minas Geraes [Gerais], B.[elo] Horizonte, xii. [19]51' 'Paratype, Tachytes fraternoides, ơ, R.M. Bohart [col.]'; 1 (\#MZSP 56814) '[Brazil], M.[inas] G.[erais], Passos, 8-14.v.[19]63, Claudinor Elias [col.]' 'Paratype, Tachytes fraternoides, $\$$, R.M. Bohart'; 1 9 (\#MZSP 56842) '[Brazil], Ypiranga [Ipiranga], E.[stado] S.[ão] Paulo' 'Paratype, Tachytes fraternoides, R.M. Bohart [col.]'.

\section{Tachytes menkei Bohart, 1978: 469}

Paratypes: $1 \sigma^{\circ}$ and 1 (\#MZSP 56811, 56812) '[Brazil], Amazonas, Vista Alegre, Rio Branco, 6.ix.[19]24' 'Paratype, Tachytes menkei, R.M. Bohart [col.]'.

\section{Tachytes pubescens Bohart, 1978: 477 (Fig. 6A-D)}

Holotype: † (\#MZSP 56816) '[Brazil, São Paulo], Araçatuba, Rio Jacarecatinga, X-61, Lane \& Rabello Col.' 'Holotype, Tachytes pubescens, ㅇ, R.M. Bohart'.

Paratypes: 1 \% (\#MZSP 56820) '[Brazil], Goiás, Spitz col., 1935/[19]36' 'Paratype, Tachytes pubencens, ㅇ, R.M. Bohart'; 1 \% (\#MZSP 56882) '[Brazil, São Paulo], Araçatuba, x.1961, J. Lane \& Rabello.' 'Paratype, Tachytes pubencens, ㅇ, R.M. Bohart'.

\section{Tachytes richardsi Bohart, 1978: 481}

Paratypes: 1 (\#MZSP 56813) 'Suriname, Zanderijsayanne, 3-6 Aug. 1964, D.C. Geijskes' 'Malaise - Trap' 'Paratype, Tachytes richardsi, \&, R.M. Bohart'; 1 \% (\#MZSP 56868) 'Suriname, Zanderijsayanne, 27-30 July 1964, D.C. Geijskes' 'Malaise - Trap' 'Paratype, Tachytes richardsi, Macho, R.M. Bohart'.

\section{Tachytes roraimae Bohart, 1978: 482}

Paratype: 1 ๆ (\#MZSP 56869) '[Brazil], Terr.[as] Roraima, Surumu, ix.1966' 'M. Alvarenga col.' 'Paratype, Tachytes roraimae, , R.M. Bohart'.

\section{Tachytes rubioi Bohart, 1978: 483}

Paratype: 1 o' (\#MZSP 56870) 'Venezuela, Aragua Ocumare De La Costa, 2 km N, 12 June 1976, A.S. Menke \& D. Vincent' 'Paratype, Tachytes rubioi, o', R.M. Bohart'.

\section{Tachytes sexdens Bohart, 1978: 488}

Paratype: 1 \& (\#MZSP 56815) 'Brazil, Para [Pará], Baker' 'Paratype, Tachytes sexdens, \$, R.M. Bohart'.

\section{Tachytes stangei Bohart, 1978: 490}

Paratypes: 1 \& (\#MZSP 61894) 'Brasil, Mato Grosso, Barra do Tapirapé, 25-27.i.1964, B. Malkin col.' 'Paratype, Tachytes stangei, ᄋ, R.M. Bohart'; 1 ơ (\#MZSP 61895) '[Brazil], Est[ado] Goyaz[Goiás], Campinas[Goiânia], 1935, R. Spitz col.' 'Paratype, Tachytes stangei, ox, R.M. Bohart'.

\section{Oxybelini \\ Oxybelus Latreille}

\section{Oxybelus cinemucro Bohart, 1993a: 20}

Paratype: 1 ơ (\#MZSP 61846) '[Argentina], Tucuman, Cafayate, 4 January, 1956' 'Paratype, Oxybelus cinemurco, $\sigma^{\prime \prime}$, R.M. Bohart'.

\section{Oxybelus genisei Bohart, 1993a: 21}

Paratypes: 1 ơ (\#MZSP 61847) '[Brazil], Sta [Santa] Catarina, Nova Teutonia, xii.1967' 'F. Plaumann Coll' 'Paratype, Oxybelus genisei, o', R.M. Bohart'; 1 ㅇ (\#MZSP 61848) 'Brazilien [Brazil], Nova Teutonia, 1-26.1964, $27^{\circ} 11^{\prime} \mathrm{B} 25^{\circ} 23 \mathrm{~L}, \quad 300$ a 500 m, Fritz Plaumann' 'Paratype, Oxybelus genisei, $\$$, R.M. Bohart'.

\section{Oxybelus irwini Bohart, 1993c: 164}

Paratype: 1 o' (\#MZSP 61849) 'Queza Itepeque, El Salvador, 23.vi.1963' 'D. Cavagnaro, M.E. Irwini, Collectors' 'Paratype, Oxybelus irwini, o’, R.M. Bohart'.

\section{Oxybelus plaumanni Bohart, 1993b: 74}

Paratypes: 1 \& (\#MZSP 61843) '[Brazil], MT [Mato Grosso], Faz.[enda] Floresta Mun.[icipal] Três Lagoas, 13-20. ix.1964, Exp.[edição] Depto.[Departamento] Zool.[ologia]' 'Paratype, Oxybelus plaumanni, , R.M. Bohart [col.]'; 1 \& (\#MZSP 61844) '[Brazil], S[ão] Paulo, Ibitinga, Faz.[enda] Itaquerê, 29.08.1964, K. Lenko

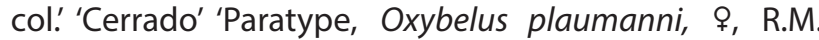
Bohart'; 1 o" (\#MZSP 61845) 'Argentina, Santiago del Estero, Los Tigres, 11-16.i.1970, P. Gelbach' 'Paratype, Oxybelus plaumanni, ơ', R.M. Bohart'. 


\section{Oxybelus roraimae Bohart, 1993b: 74}

Paratypes: $1 \sigma^{\prime}$ and 1 (\#MZSP 61839, 61840) '[Brazil], Surumu, Terr.[a] Roraima, ix.1966' 'M. Alvarenga Col.' 'Paratype, Oxybelus roraimae, R.M. Bohart'.

\section{Oxybelus schusteri Bohart, 1993a: 22}

Paratype: 1 \& (\#MZSP 61841) '[Ecuador], Galápagos, Darwin Res[earch] Sta[tion] Santa Cruz, 20.ii.1964' 'R.O. Schuster Collector' 'Paratype, Oxybelus schusteri, ㅇ, R.M. Bohart'.

\section{Oxybelus stangei Bohart, 1993a: 24}

Paratype: 1 o' (\#MZSP 61842) ‘[Brazil], S.[anta] Catharina [Catarina], Nova Teutonia, October, 1974' 'F. Plaumann, BM 1975-174 n०1' 'Paratype, Oxybelus stangei, o', R.M. Bohart'.

\section{Trypoxylini Trypoxylon Latreille}

Trypoxylon anapaike Amarante, 1991: 431 (Fig. 7A-D)

Holotype: $q$ (\#MZSP 61893) ' Suriname, Anapaike, (Rio Lawa), Marowijne distr., xi.1963, B. Malkin' 'Holotipo' 'Trypoxylon anapaike, ㅇ, Amarante, 1991, holótipo, S.T.P. Amarante, det. 1991'.

\section{Trypoxylon latro Menke \& Richards, 1983: 152}

Paratype: 1 (\#MZSP 61896) 'Costa Rica, Puntarenas Prov.[incia], Corcovado Nat.[ional] Pk.[Park], Sirena Station, iii.1981, R.W. Matthews' 'NEST C' 'Trypoxylon latro, Richards, + , paratype' 'Paratypus'.

\section{Trypoxylon lenkoi Amarante, 1991: 433 (Fig. 8A-D)}

Holotype: ơ (\#MZSP 61850) '[Brazil], S[ão] $\mathrm{P}$ [aulo], Faz.[enda] Itaquerê, Bôa Esperança do Sul, 21.vii.1964, K. Lenko col.' 'KL. 72' 'Holotipo', 'Trypoxylon lenkoi, o', Amarante, 1991, holótipo S.T.P. Amarante, det. 1991'.

Paratype: 1 ơ (\#MZSP 61851) '[Brazil], S[ão] P[aulo], Faz.[enda] Itaquerê, Bôa Esperança do Sul, 21.vii.1964, K. Lenko col.' 'KL. 72' 'Paratipo', 'Trypoxylon lenkoi, ơ', Amarante, 1991 Parátipo, S.T.P. Amarante, det. 1991'.

\section{Trypoxylon mojuba Amarante, 1995: 157 (Fig. 9A-D)}

Holotype: \& (\#MZSP 61852) 'Brasil, AM[azonas], Manaus, Faz.[enda] Esteio, 59 $43^{\prime} 40^{\prime \prime} \mathrm{W}$; 02 $24^{\prime} 26^{\prime}\left[{ }^{\prime \prime}\right]$ S, 5.iv.1990, E.F. Morato col.' 'INPA/WWF PDBFF 1501c/ 1854/3' 'HOLÓTIPO', 'Trypoxylon mojuba, \&, Amarante, 1995, Holótipo, S.T.P. Amarante det. 1995'.
Trypoxylon personatum Amarante, 1991: 430 (Fig. 10A-D)

Holotype: $\sigma^{\top}$ (\#MZSP 61956)'Brasil, AM[azonas], Remanso Rio Madeira, 8.xii.1975, Exp.[edição] Perm.[anente da] Am.[azônia]' 'HOLOTIPO', 'Trypoxylon personatum, o', Amarante, 1991 holótipo, S.T.P. Amarante det. 1991'.

\section{Pemphredoninae \\ Pemphredonini \\ Microstigmus Ducke}

\section{Microstigmus brasiliensis Melo, 1992: 666 (Fig. 11A-D)}

Holotype: $q$ (\#MZSP 61856) 'Brasil, M[inas] G[erais], Viçosa, 10.iv.1989, G.A.R. MELO' 'HOLOTYPUS, \&, Microstigmus brasiliensis, G.A.R. MELO 1992'.

Paratypes: 1 o' (\#MZSP 61857) 'Brasil, M[inas] G[erais], Viçosa, 06.viii.1989, G.A.R. Melo' 'Paratype, Microstigmus brasiliensis, o', G.A.R. Melo 1992'; 1 o' (\#MZSP 61858)'Brasil, M[inas] G[erais], Viçosa, 28.i.1990, G.A.R. Melo' 'Paratype, Microstigmus brasiliensis, o', G.A.R. Melo 1992'; 1 \% (\#MZSP 61859) ‘Brasil, M[inas] G[erais], Viçosa, 19.viii.1988, G.A.R. Melo' 'Paratype, Microstigmus brasiliensis, \&, G.A.R. Melo 1992'.

\section{Microstigmus cooperi Melo \& Matthews, 1997: 422} (Fig. 12A-D)

Holotype: 9 (\#MZSP 61853) 'Brasil, Amazonas, 60 km ao norte de Manaus, 13.IX.1991, G.A.R. Melo' 'Faz. [enda] Esteio $\left(02^{\circ} 25^{\prime} \mathrm{S}\right.$; 59 $\left.50^{\circ} \mathrm{W}\right)$, Res. 1501 (PDBFF), Ninho 449' 'HOLOTYPE, Microstigmus cooperi, Melo and Matthews'.

Paratypes: 1 \% and $1 \sigma^{\prime}$ (\#MZSP 61854, 61855) 'Brasil, Amazonas, $60 \mathrm{~km}$ ao norte de Manaus, 13.ix.1991, G.A.R. Melo' 'Faz.[enda] Esteio (02²5'S; 59 $\left.50^{\circ} \mathrm{W}\right)$, Res. 1501 (PDBFF), Ninho 449' 'Paratype, Microstigmus cooperi, Melo and Matthews'.

\section{Microstigmus flavus Melo \& Matthews, 1997: 428} (Fig. 13A-D)

Holotype: 9 (\#MZSP 61860) 'Brasil, Minas Gerais, Viçosa, 8.XII.1992, G.A.R. Melo (N645)' 'HOLOTYPE, Microstigmus flavus, Melo and Matthews'.

Paratypes: $1 \sigma^{7}$ (\#MZUSP 61861) 'Brasil, Minas Gerais, Viçosa, 5.ii.1989, G.A.R. Melo (N188)' 'Paratype, Microstigmus flavus, Melo and Matthews'; 1 \% (\#MZUSP 61862) 'Brasil, Minas Gerais, Marliéria, 24.ix.1989, G.A.R. Melo (N304)' 'Paratype, Microstigmus flavus, Melo and Matthews'; 1 9 (\#MZSP 61863) 'Brasil, Minas Gerais, G.[overnador] Valadares, 19.vii.1991, G. Melo (N397)' 'Paratype, Microstigmus flavus, Melo and Matthews'. 


\section{Microstigmus nigrophthalmus Melo, 1992: 663 (Fig. 14A-D)}

Holotype: ? (\#MZSP 61864) 'Brasil, Minas Gerais, Viçosa, 06.viii.1989, G.A.R. MELO' 'HOLOTYPUS Microstigmus nigrophthalmus, , G.A.R. MELO, 1992'.

Paratypes: 1 (\#MZSP 61865) 'Brasil, M[inas] G[erais], Araponga, 13.v.1989, G.A.R. Melo' 'Paratype, Microstigmus nigrophthalmus, \&, G.A.R. Melo, 1992'; 1 @ (\#MZSP 61866) 'Brasil, M[inas] G[erais], Viçosa, 21.iv.1988, G.A.R. Melo' 'Paratype, Microstigmus nigrophthalmus, ㅇ, G.A.R. Melo, 1992'; 1 우 (\#MZUSP 61867) 'Brasil, M[inas] G[erais], Viçosa, 24.iv.1988, G.A.R. Melo' 'Paratype, Microstigmus nigrophthalmus,,+ G.A.R. Melo, 1992'; 1 o (\#MZSP 61868) 'Brasil, M[inas] G[erais], Viçosa, 09.iv.1988, G.A.R. Melo' 'Paratype, Microstigmus nigrophthalmus, \&, G.A.R. Melo, 1992'; 1 o' (\#MZSP 61869) 'Brasil, M[inas] G[erais], Viçosa, 17.iv.1988, G.A.R. Melo' 'Paratype, Microstigmus nigrophthalmus, o', G.A.R. Melo, 1992'; 1 ơ (\#MZSP 61870) 'Brasil, M[inas] G[erais], Viçosa, 10.viii.1988, G.A.R. Melo' 'Paratype, Microstigmus nigrophthalmus, o", G.A.R. Melo, 1992'; 1 o" (\#MZSP 61871) 'Brasil, M[inas] G[erais], Viçosa, 13.xi.1988, G.A.R. Melo' 'Paratype, Microstigmus nigrophthalmus, o', G.A.R. Melo, 1992'; 1 o' (\#MZSP 61872) 'Brasil, M[inas] G[erais], Viçosa, 05.ii.1989, G.A.R. Melo' 'Paratype, Microstigmus nigrophthalmus, o", G.A.R. Melo, 1992'; 1 o" (\#MZSP 61873) 'Brasil, M[inas] G[erais], Viçosa, 3.ii.1989, G.A.R. Melo' 'Paratype, Microstigmus nigrophthalmus, o', G.A.R. Melo, 1992'; 1 o' (\#MZSP 61874) 'Brasil, M[inas] G[erais], Viçosa, 18.v.1989, G.A.R. Melo' 'Paratype, Microstigmus nigrophthalmus, o", G.A.R. Melo, 1992'; 1 o' (\#MZSP 61875) 'Brasil, M[inas] G[erais], Viçosa, 1.v.1989, G.A.R. Melo' 'Paratype, Microstigmus nigrophthalmus, o', G.A.R. Melo, 1992'; 1 ơ (\#MZSP 61876) 'Brasil, M[inas] G[erais], Viçosa, 15.ix.1888, G.A.R. Melo' 'Paratype, Microstigmus nigrophthalmus, o", G.A.R. Melo, 1992'; 1 o" (\#MZSP 61877) 'Brasil, M[inas] G[erais], Viçosa, 9.vii.1989, G.A.R. Melo' 'Paratype, Microstigmus nigrophthalmus, o', G.A.R. Melo, 1992'; 1 o' (\#MZSP 61878) 'Brasil, M[inas] G[erais], Viçosa, 29.xi.1989, G.A.R. Melo' 'Paratype, Microstigmus nigrophthalmus, o", G.A.R. Melo, 1992'; 1 o' (\#MZSP 61879) 'Brasil, M[inas] G[erais], Viçosa, 1.vi.1989, G.A.R. Melo' 'Paratype, Microstigmus nigrophthalmus, o', G.A.R. Melo, 1992'; 2 o' (\#MZSP 61880, 61881) 'Brasil, M[inas] G[erais], Viçosa, 17.iv.1988, G.A.R. Melo' 'Paratype, Microstigmus nigrophthalmus, o', G.A.R. Melo, 1992'; 1 o' (\#MZSP 61882) ‘Brasil, M[inas] G[erais], Viçosa, 9.iv.1989, G.A.R. Melo' 'Paratype, Microstigmus nigrophthalmus, o', G.A.R. Melo, 1992'.

\section{Microstigmus similis Melo, 1993 (in Melo \& Evans, 1993): 261 (Fig. 15A-D)}

Holotype: 우 (\#MZSP 61883) 'Brasil, M[inas] G[erais], Viçosa, 29.xi.1991, G. MELO \& M. COSTA [Ninho em orifício em esteio. Mata do Paraíso (Casa do Sr. R. Stanciola)]' 'HOLOTYPUS $\$$, Microstigmus similis, G.A.R. MELO 1992'.
Paratype: 1 \% (\#MZSP 61884) 'Brasil, M[inas] G[erais], Viçosa, 29.11.1991, G. Melo \& M. Costa' 'Paratype, Microstigmus similis, \&, G.A.R. Melo 1992'.

Comments: The original publication reports 2 females paratypes deposited in the MZSP, but only one was located.

\section{Microstigmus xanthosceles Melo \& Matthews, 1997: 434}

Paratype: 1 ㅇ (\#MZSP 61885) 'Costa Rica, Guanacaste Pv[Province], Sta. [Santa] Rosa NP, Bosq.[e] Hum[edo]11-O' 'Janzen \& Gauld, 18.i-8.ii.1986' 'Paratype, Microstigmus xanthosceles, Melo and Matthews'.

\section{Microstigmus xylicola Melo, 1993 (in Melo \& Evans, 1993): 258 (Fig. 16A-D)}

Holotype: \& (\#MZSP 61886) 'Brasil, M[inas] G[erais], Viçosa, [Ninho 1: 3 \& (1 pre-p.; 1 p. de parasita). Telhado de cisterna], 24.xi.1990, G.A.R. MELO' 'HOLOTYPUS \&, Microstigmus xylicola, G.A.R. MELO 1992'.

Paratypes: 4 을 $2 \sigma^{\prime}$ (\#MZSP 61887 - 61892) 'Brasil, M[inas] G[erais], Viçosa, 24.xi.1990, G.A.R. Melo' 'Paratype, Microstigmus xylicola, G.A.R. Melo 1992'.

\section{Sphecidae \\ Sphecinae \\ Sphecini \\ Sphex Linnaeus}

\section{Sphex subhyalinus Fox, 1899: 199 (Fig. 17A-D)}

Holotype: ㅇ (\#MZSP 61647) '[Brazil], E[stado] S[ão] P[aulo], Ypiranga [Ipiranga]' 'Sphex subhyalinna, Fox det. Type!' 'Sphex subhyalinus, Type Fox'.

\section{ACKNOWLEDGMENTS}

This work was supported by grants from Conselho Nacional de Desenvolvimento Científico e Tecnológico, CNPq (440574/2015-3) and Fundação de Amparo à Pesquisa do Estado de São Paulo, FAPESP (2016/503788 and 17/07366-1) to CRFB. TOA and ADS are grateful to PROTAX/CNPQ for the fellowships (processes 152579/2016-8 and 134124/2016-2, respectively). HCO and KSR thanks Coordenação de Aperfeiçoamento de Pessoal de Nível Superior - Brasil (CAPES finance codes $88887.136354 / 2017-00$ and $1798335 / 2018)$ for the postdoctoral grants. CRFB thanks both agencies for continuous support.

Authors' contributions: All authors contributed equally for this work. 


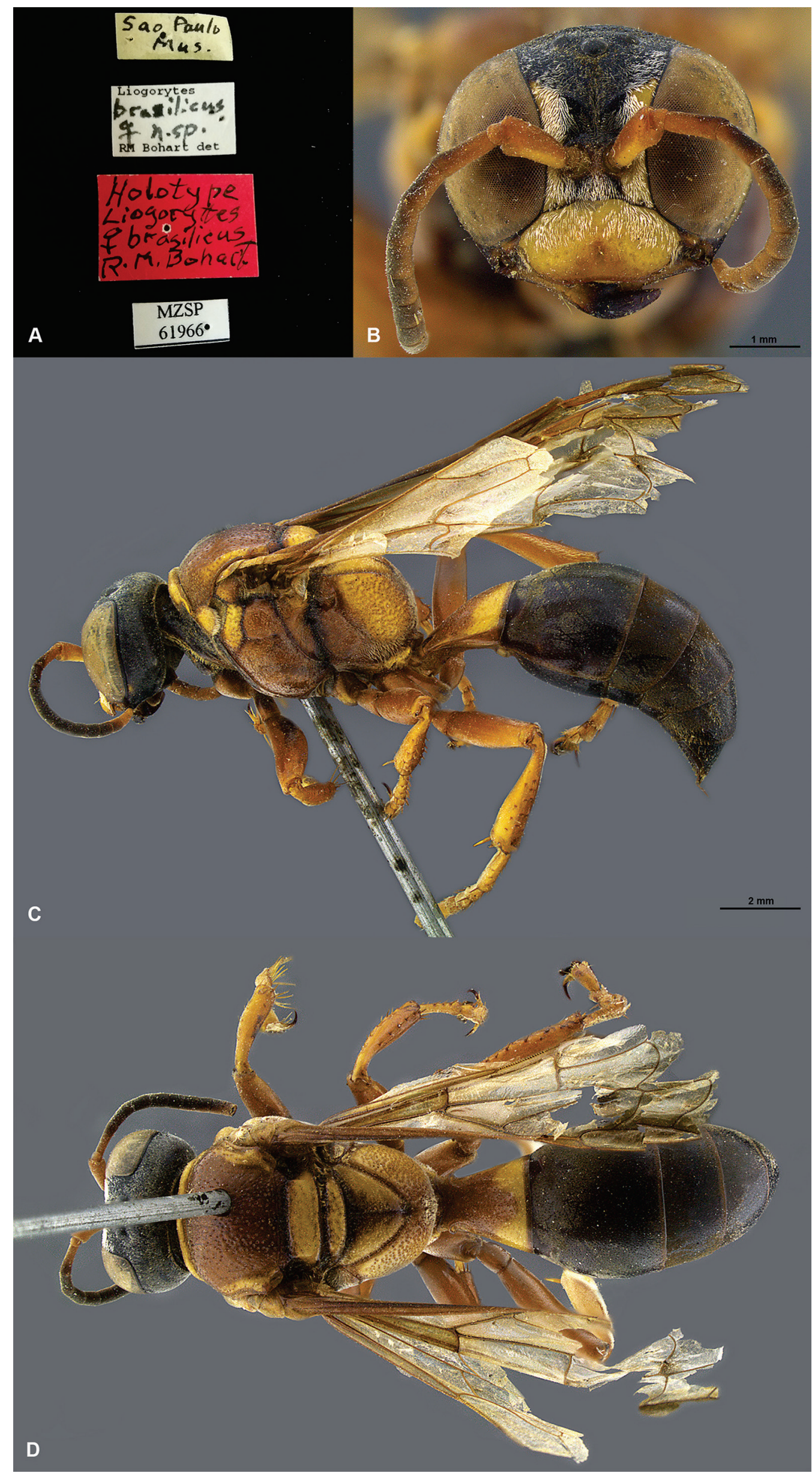

Figure 1. Holotype female of Liogorytes brasilicus Bohart, 2000. (A) labels. (B) head in frontal view. (C) body in lateral view. (D) body in dorsal view. 


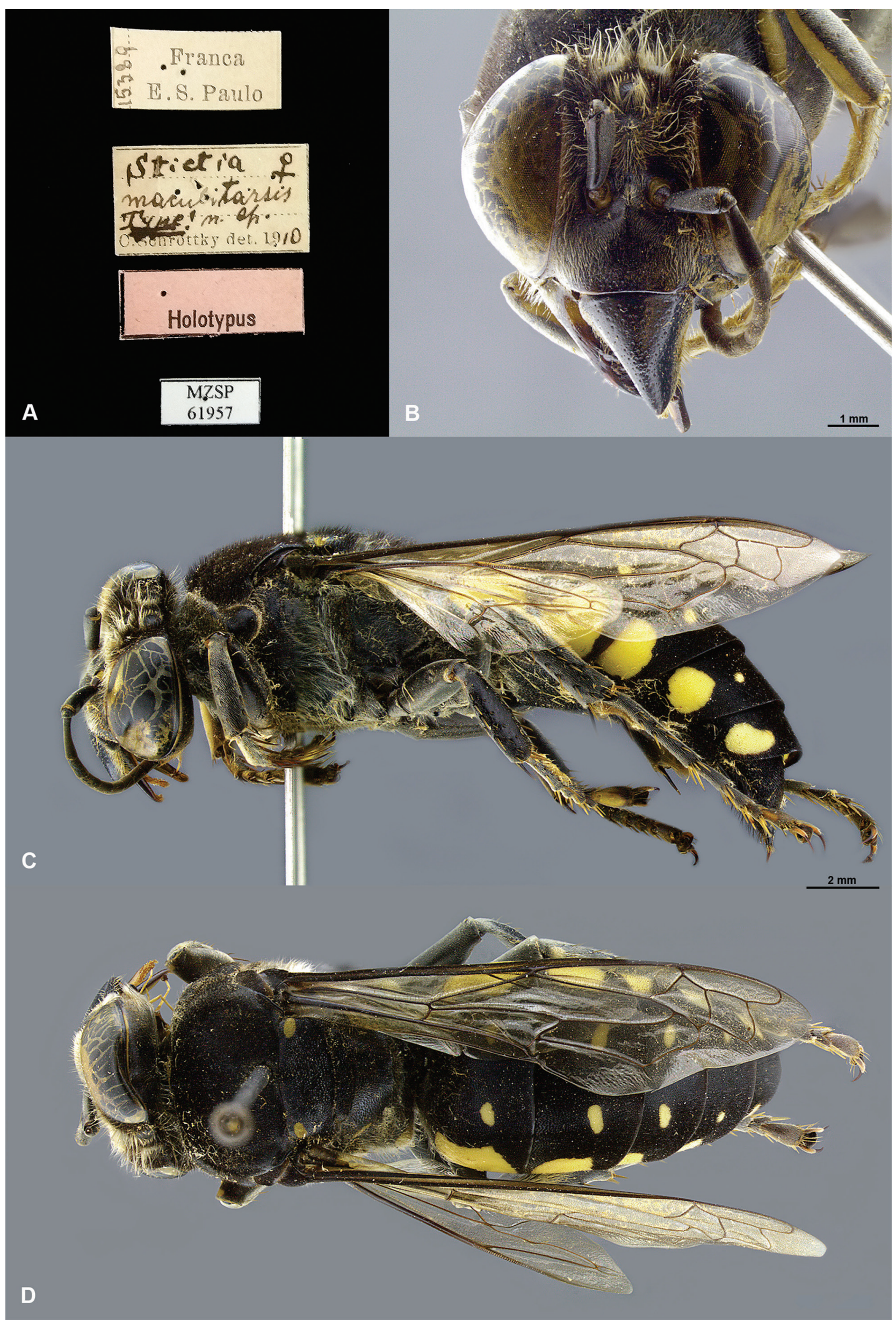

Figure 2. Holotype female of Stictia maculitarsis Schrottky, 1913. (A) labels. (B) head in frontal view. (C) body in lateral view. (D) body in dorsal view. 

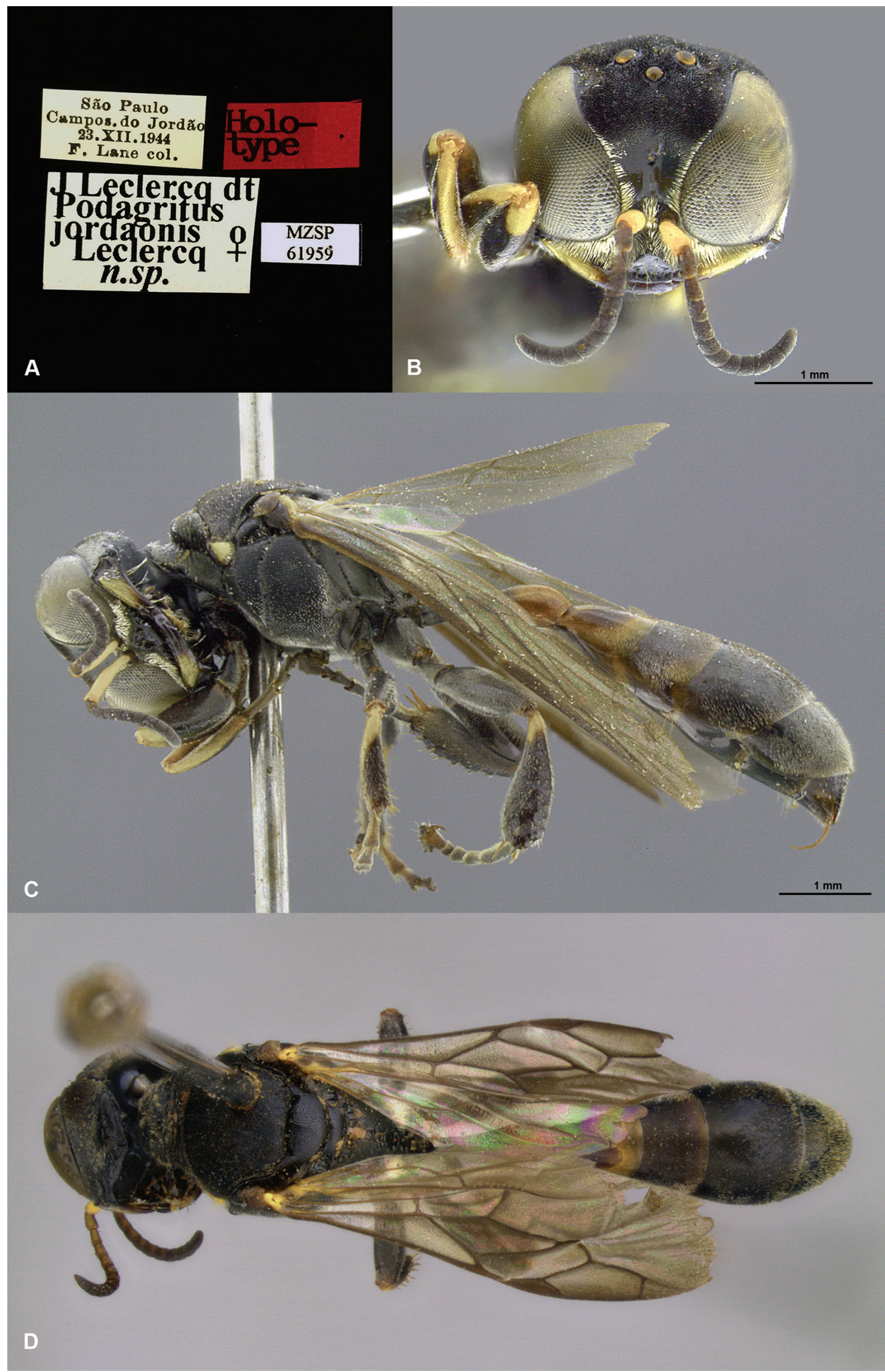

Figure 3. Holotype female of Podagritus jordaonis Leclercq, 2000. (A) labels. (B) head in frontal view. (C) body in lateral view. (D) body in dorsal view. 


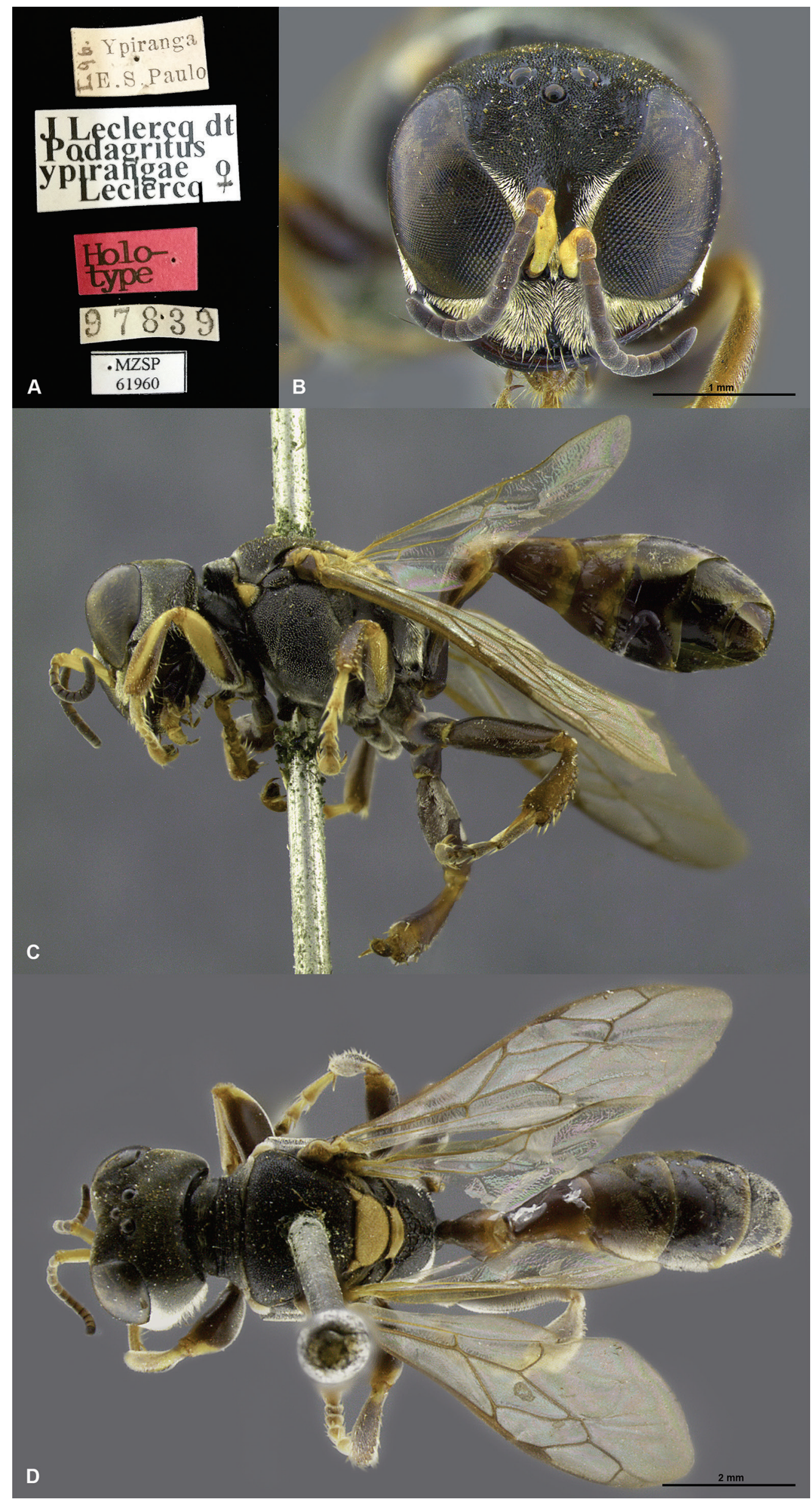

Figure 4. Holotype female of Podagritus ypirangae Leclercq, 2000. (A) labels. (B) head in frontal view. (C) body in lateral view. (D) body in dorsal view. 


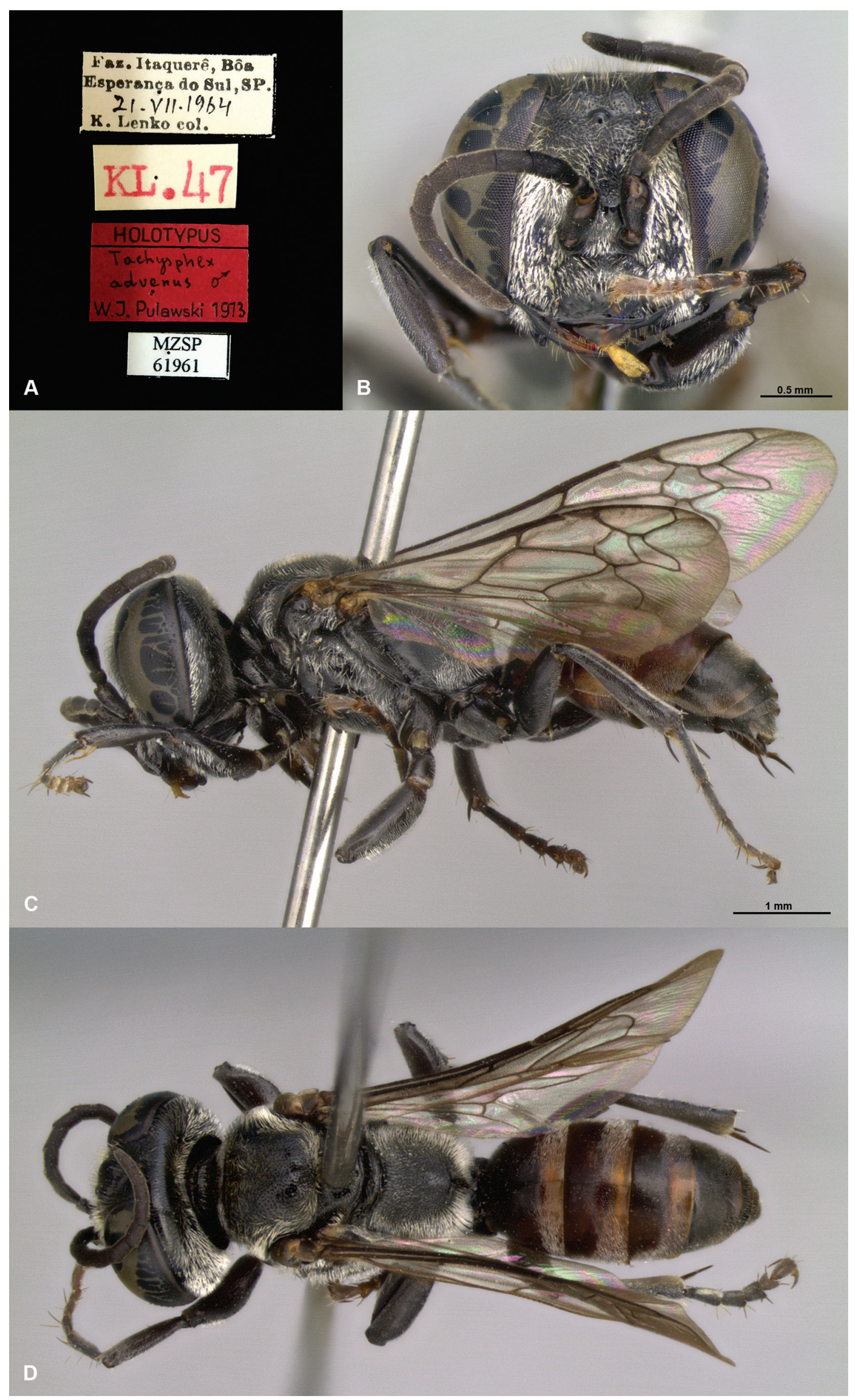

Figure 5. Holotype male of Tachysphex advenus Pulawski, 1974. (A) labels. (B) head in frontal view. (C) body in lateral view. (D) body in dorsal view. 


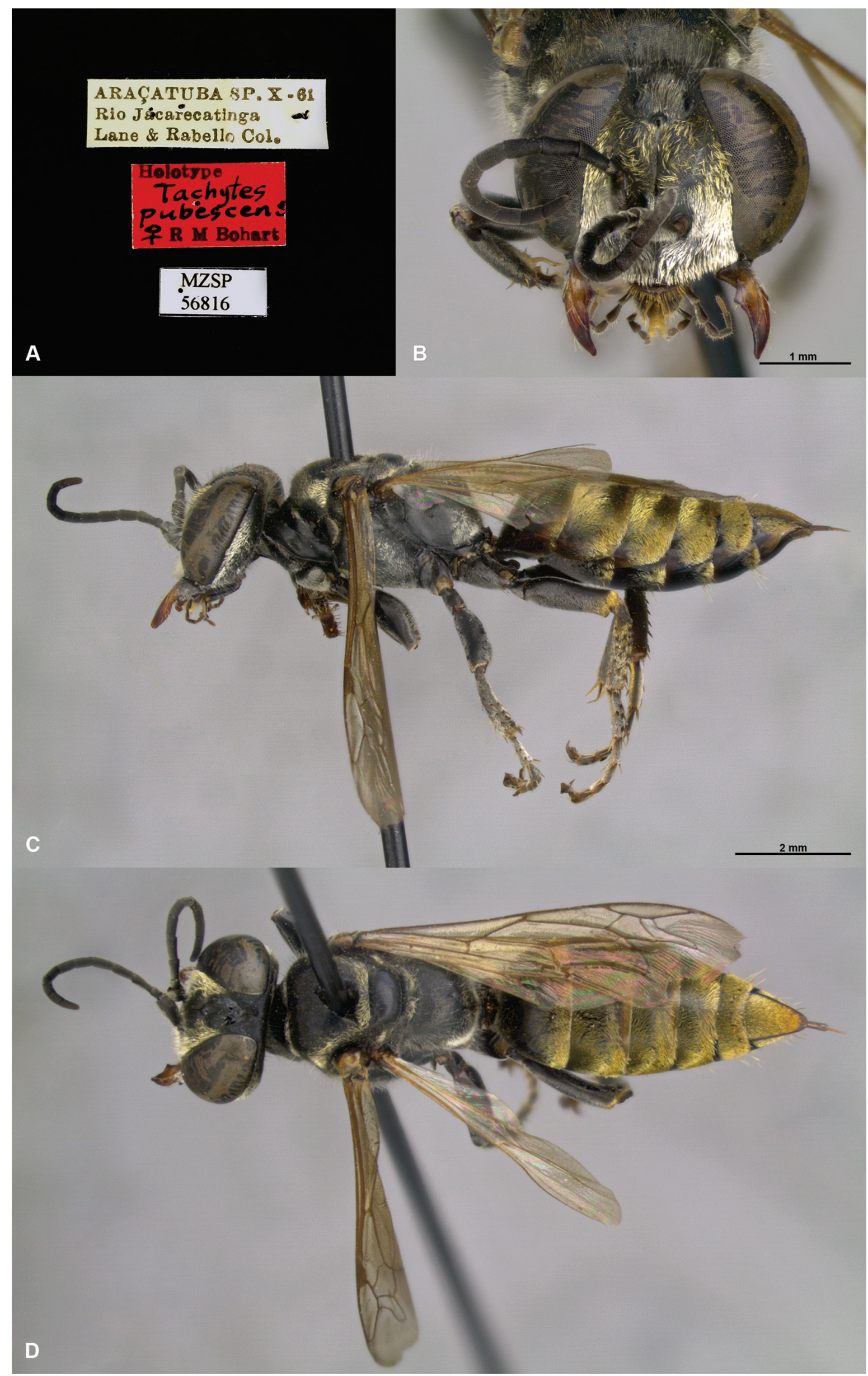

Figure 6. Holotype female of Tachytes pubescens Bohart, 1979. (A) labels. (B) head in frontal view. (C) body in lateral view. (D) body in dorsal view. 

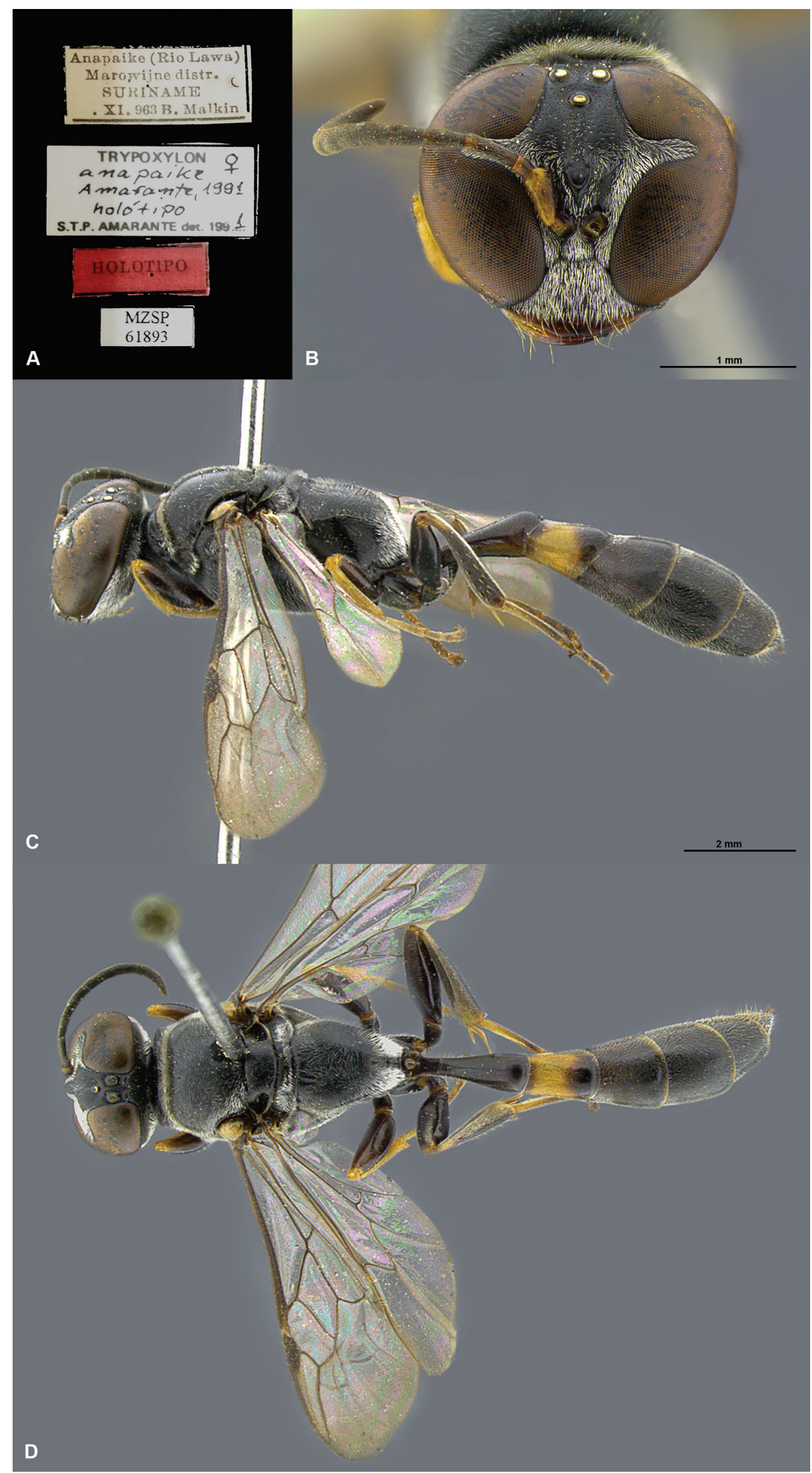

Figure 7. Holotype female of Trypoxylon anapaike Amarante, 1991. (A) labels. (B) head in frontal view. (C) body in lateral view. (D) body in dorsal view. 


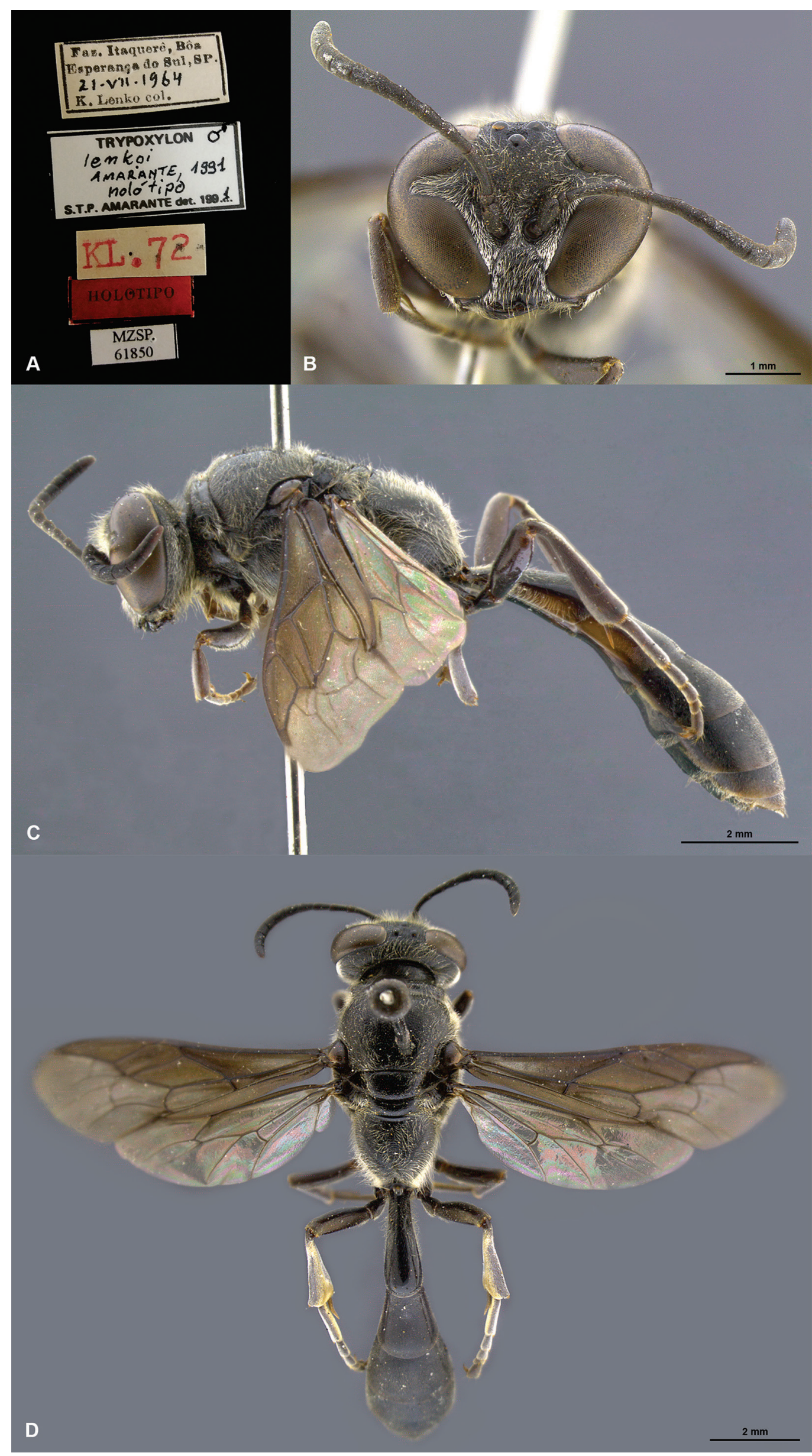

Figure 8. Holotype male of Trypoxylon lenkoi Amarante, 1991. (A) labels. (B) head in frontal view. (C) body in lateral view. (D) body in dorsal view. 

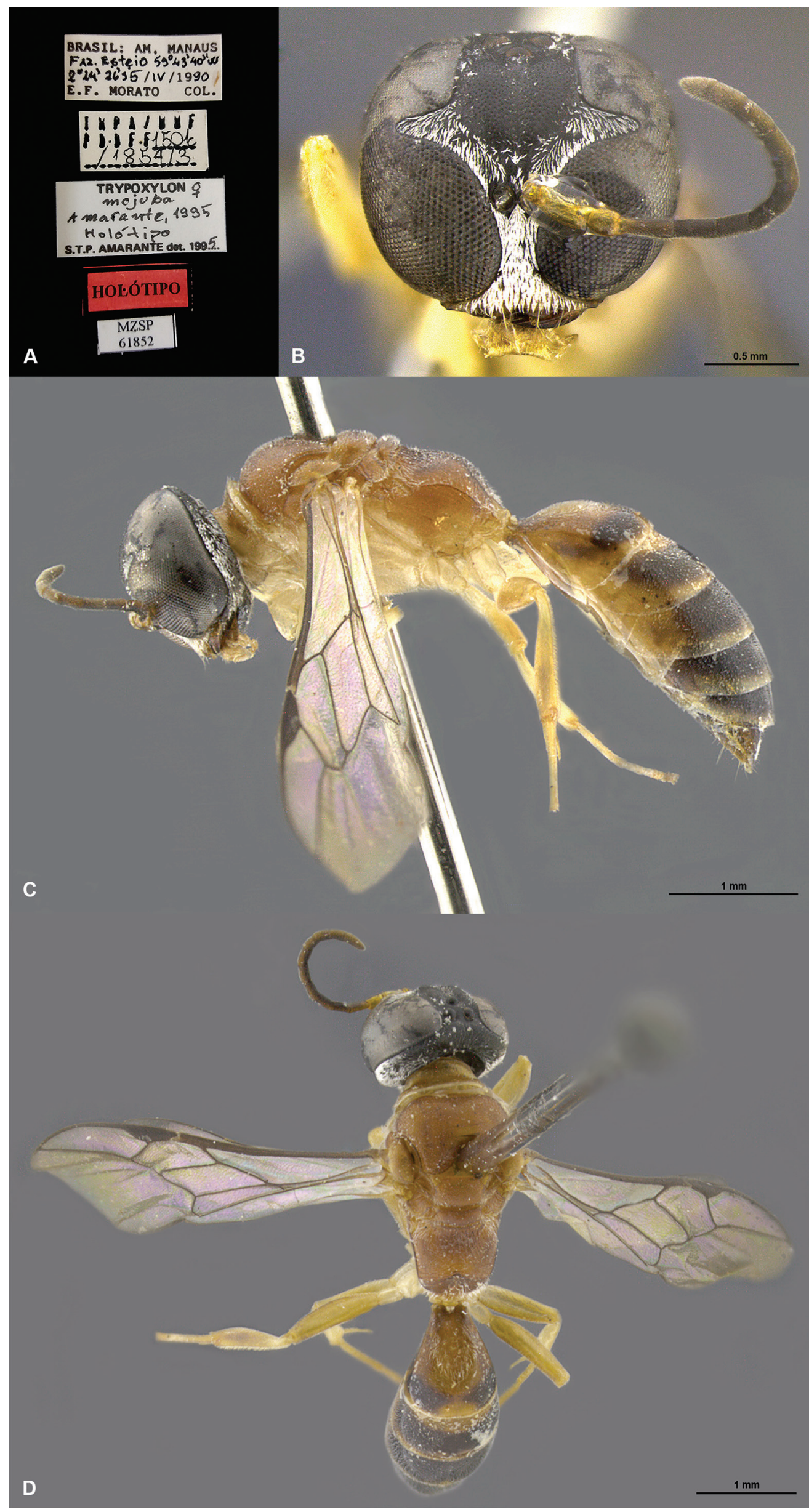

Figure 9. Holotype female of Trypoxylon mojuba Amarante, 1995. (A) labels. (B) head in frontal view. (C) body in lateral view. (D) body in dorsal view. 


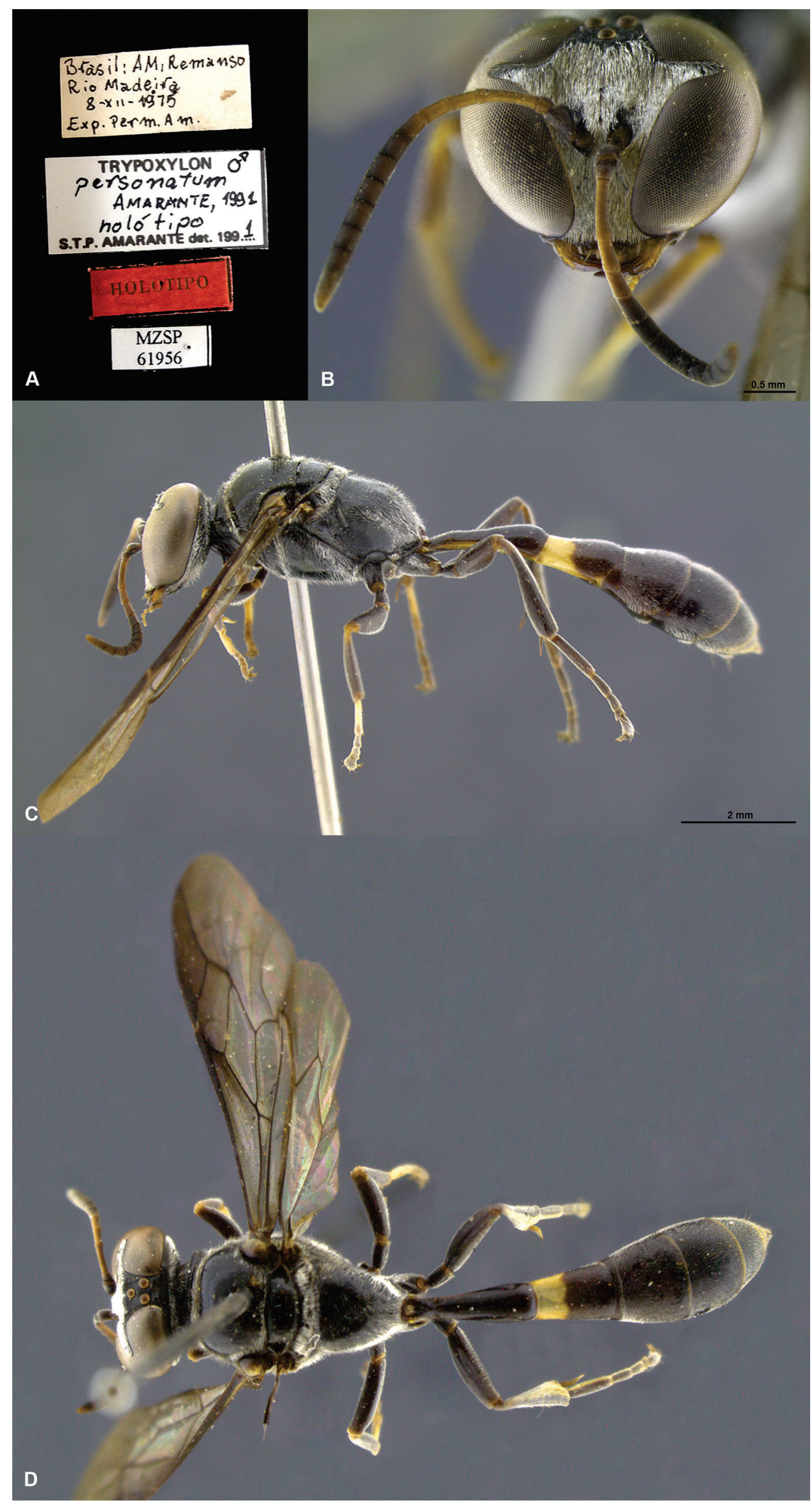

Figure 10. Holotype male of Trypoxylon personatum Amarante, 1991. (A) labels. (B) head in frontal view. (C) body in lateral view. (D) body in dorsal view. 

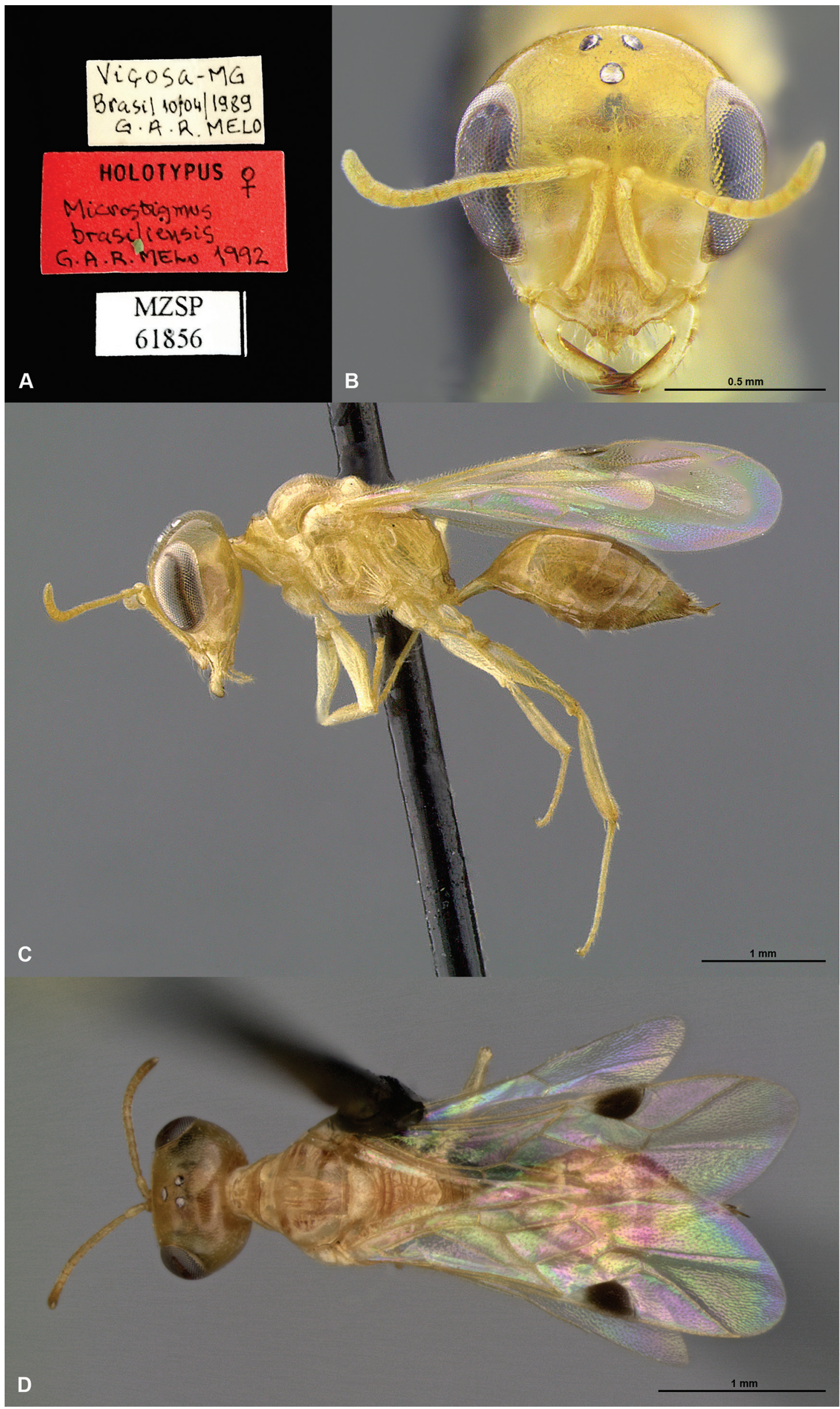

Figure 11. Holotype female of Microstigmus brasiliensis Melo, 1992. (A) labels. (B) head in frontal view. (C) body in lateral view. (D) body in dorsal view. 


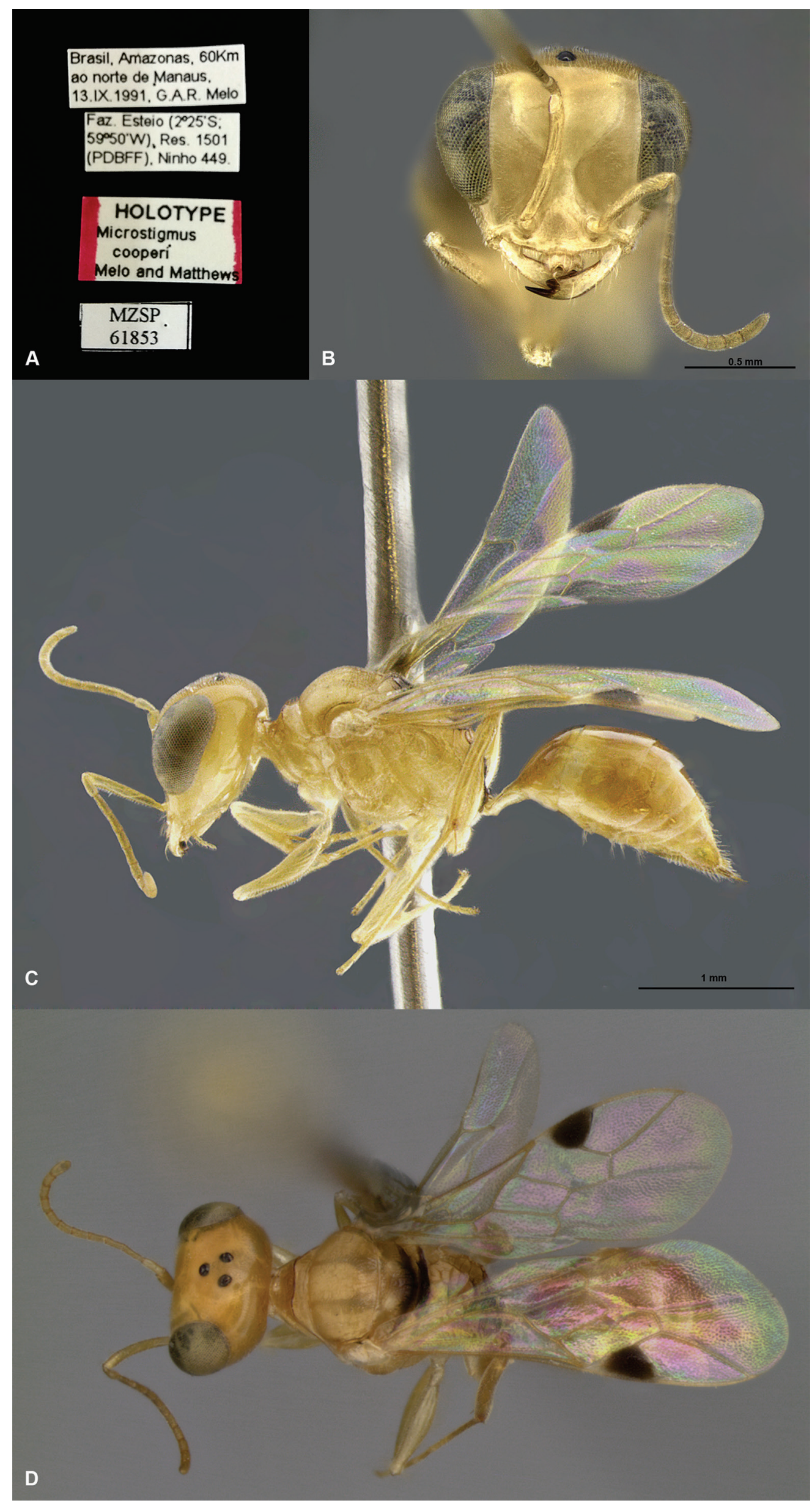

Figure 12. Holotype female of Microstigmus cooperi Melo \& Matthews, 1997. (A) labels. (B) head in frontal view. (C) body in lateral view. (D) body in dorsal view. 

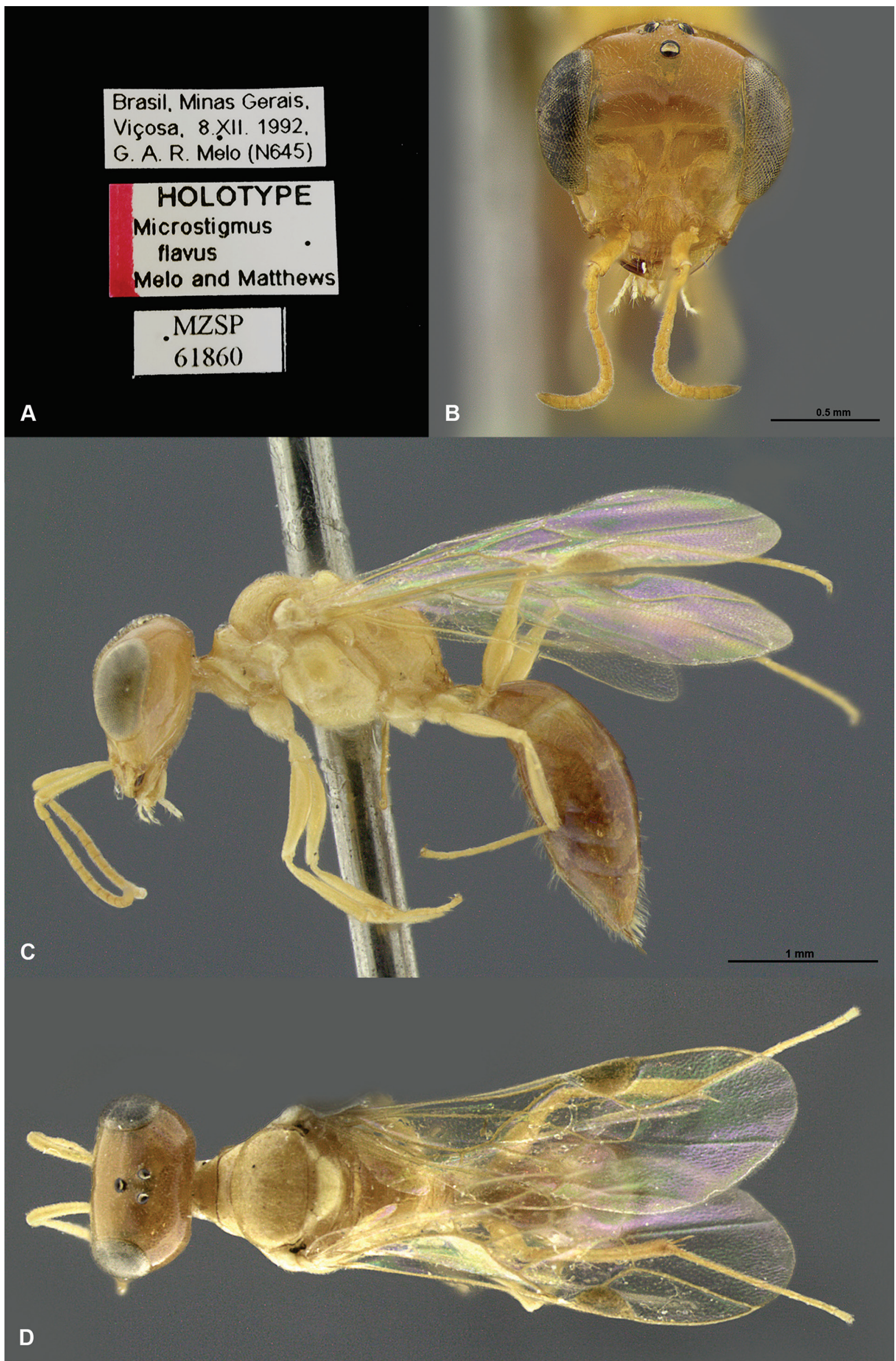

Figure 13. Holotype female of Microstigmus flavus Melo \& Matthews, 1997. (A) labels. (B) head in frontal view. (C) body in lateral view. (D) body in dorsal view. 


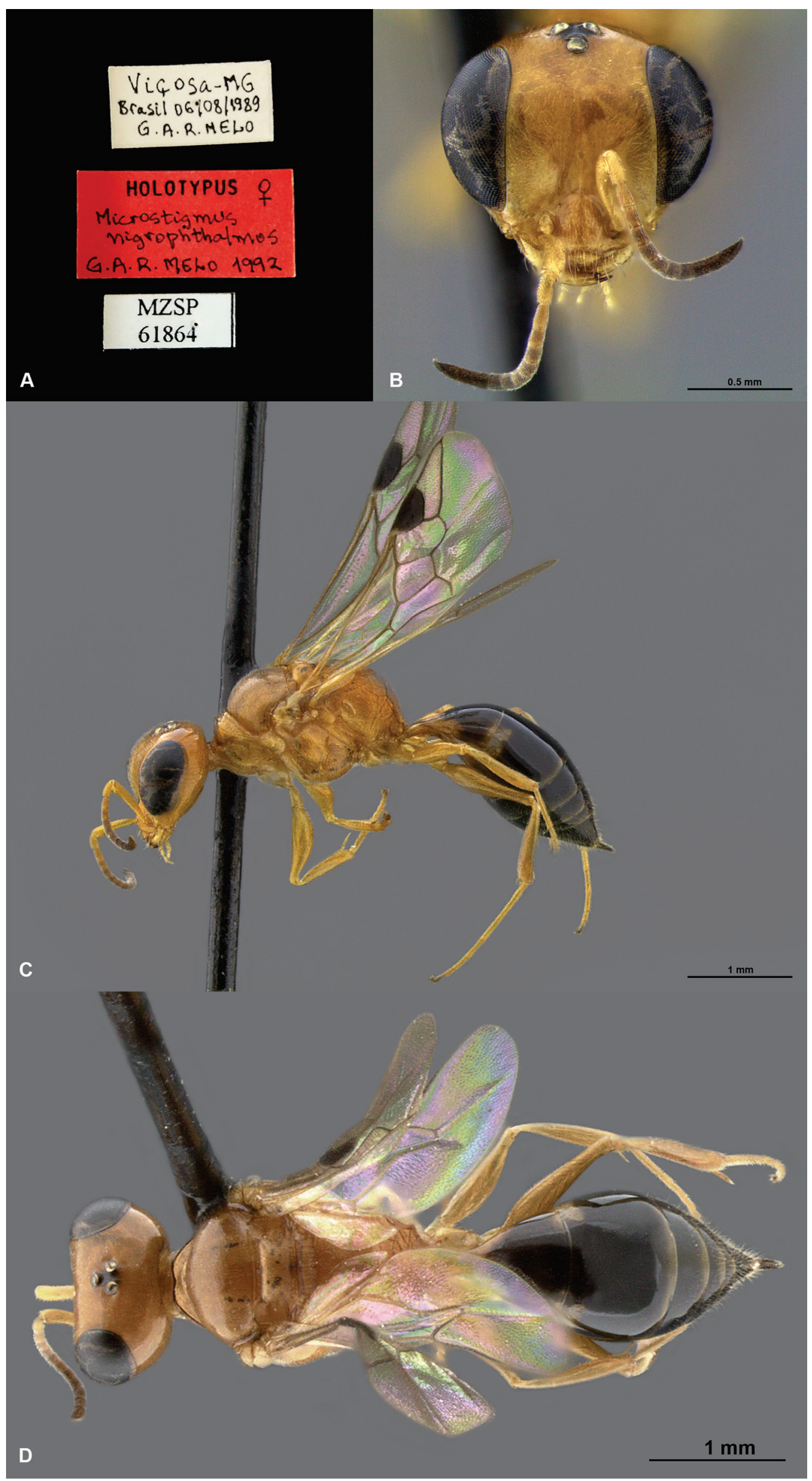

Figure 14. Holotype female of Microstigmus nigrophthalmus Melo, 1992. (A) labels. (B) head in frontal view. (C) body in lateral view. (D) body in dorsal view. 

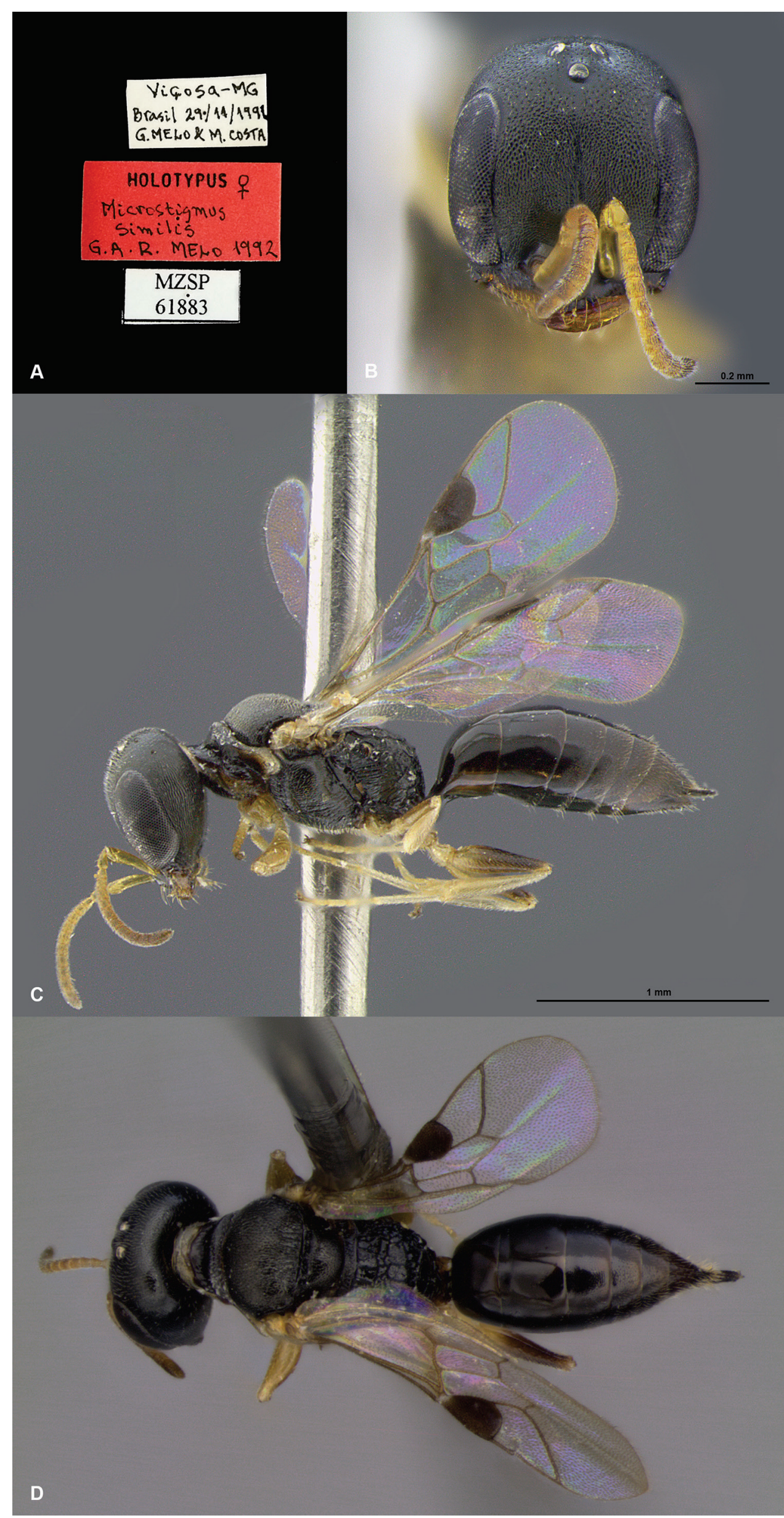

Figure 15. Holotype female of Microstigmus similis Melo, 1993. (A) labels. (B) head in frontal view. (C) body in lateral view. (D) body in dorsal view. 


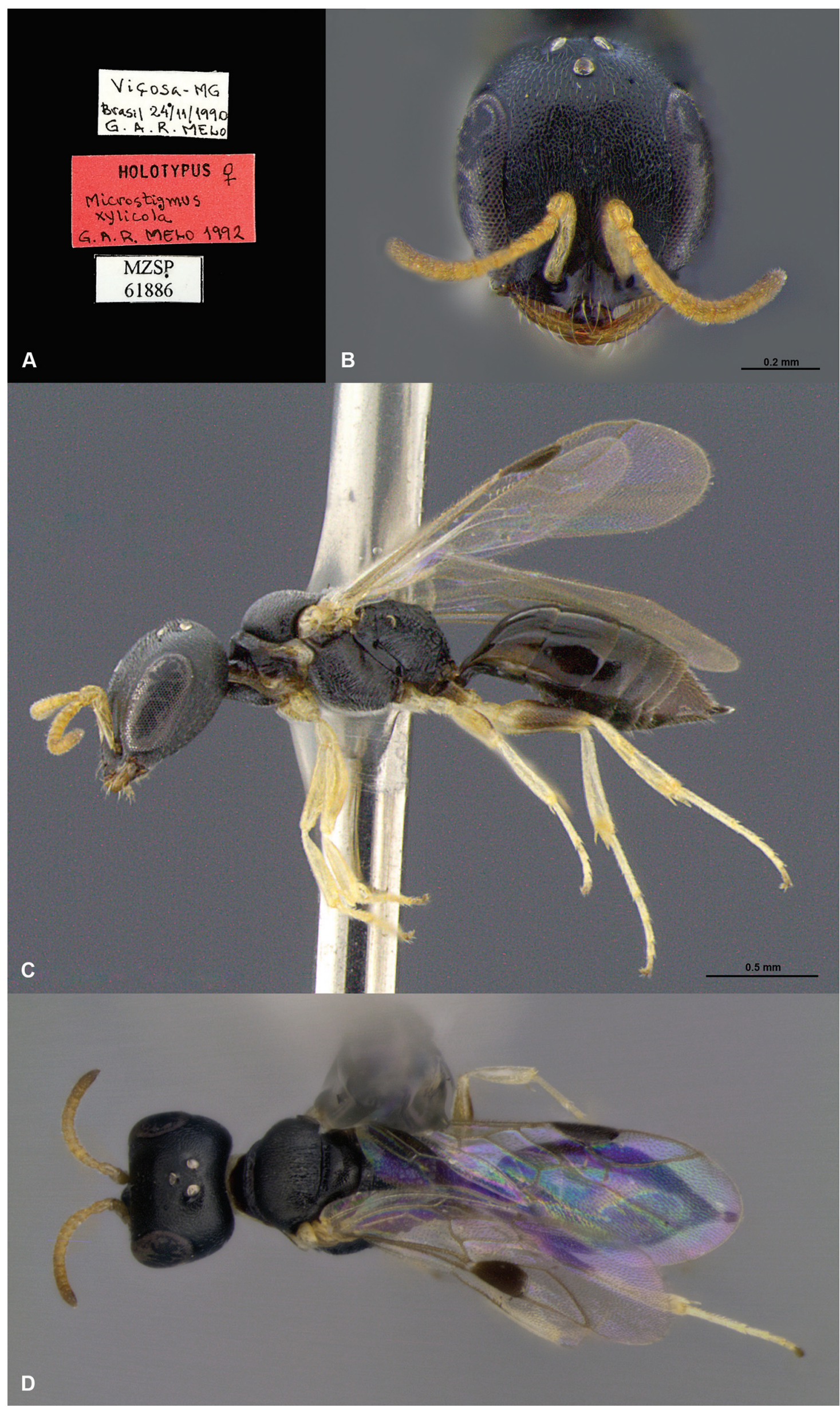

Figure 16. Holotype female of Microstigmus xylicola Melo, 1993. (A) labels. (B) head in frontal view. (C) body in lateral view. (D) body in dorsal view. 


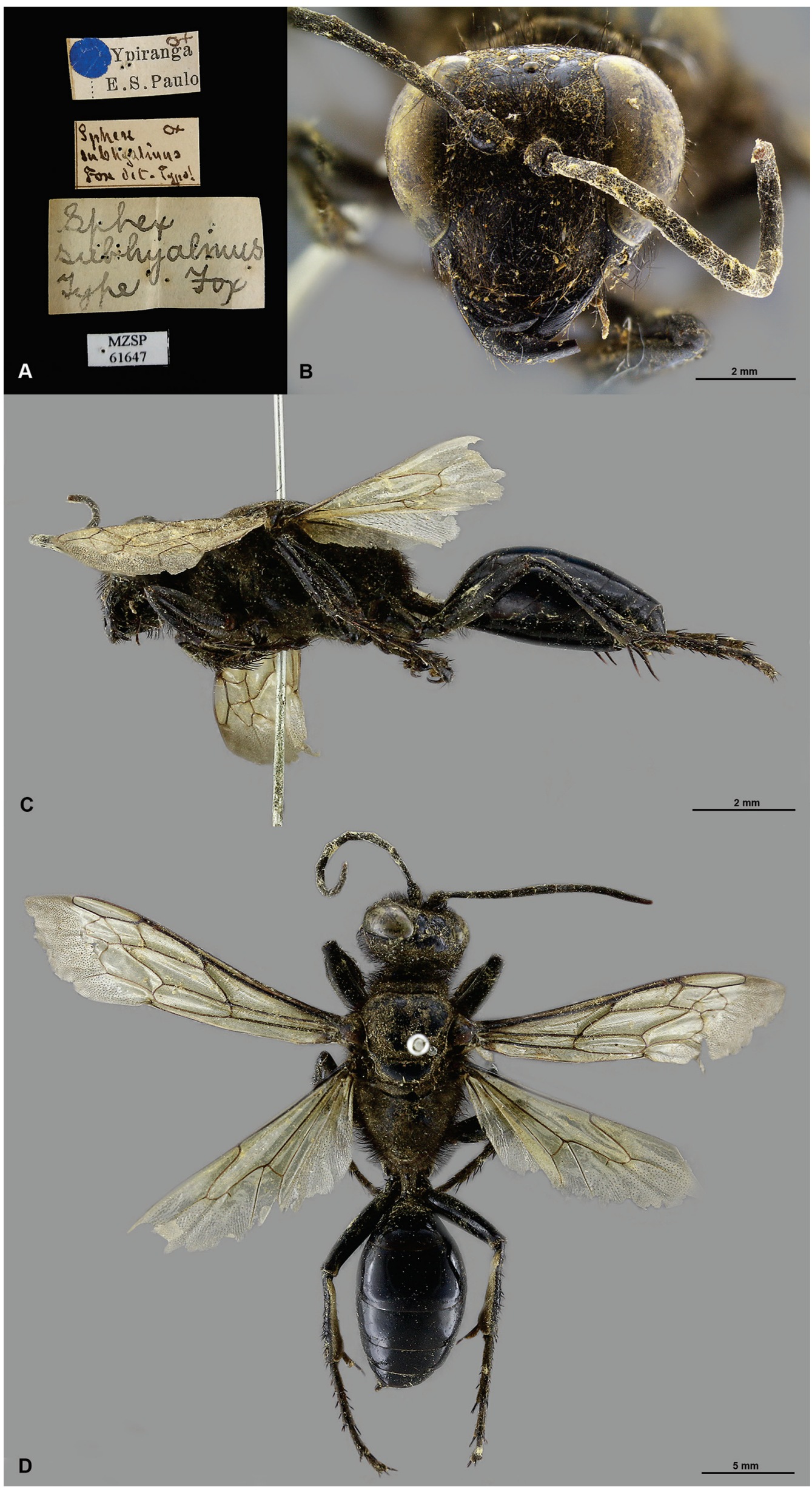

Figure 17. Holotype female of Sphex subhyalinus Fox, 1899. (A) labels. (B) head in frontal view. (C) body in lateral view. (D) body in dorsal view. 


\section{REFERENCES}

Amarante, S.T.P. 1991. Three new Neotropical species of Trypoxylon (Trypargilum) (Hymenoptera, Sphecidae). Revista Brasileira de Entomologia, 35(2): 429-437.

Amarante, S.T.P. 1995. Trypoxylon (Trypoxylon) mojuba sp. n., an unusual Trypoxylini from South America (Hymenoptera, Sphecidae). Revista Brasileira de Entomologia, 39(1): 157-160.

Amarante, S.T.P. 2006. Familia Crabronidae. In: Fernández, F. \& Sharkey, M.J. (Eds.) Introducción a los Hymenoptera de la Región Neotropical. Bogotá D.C., Sociedad Colombiana de Entomología y Universidad Nacional de Colombia. p. 457-470.

Andrade, T.; Ramos, K.; Onody, H.; Santos, A. \& Brandão, C.R.F. 2018. Type specimens of Pompiloidea, Thynnoidea and Vespoidea (Hymenoptera) deposited in the Museu de Zoologia da Universidade de São Paulo, Brazil. Papéis Avulsos de Zoologia, 58(39): 1-21. D01

Bohart, R.M. 1978. Tachytes of South America (Hymenoptera, Sphecidae, Larrinae). Transactions of the American Entomological Society, 104(3-4): 435-505.

Bohart, R.M. 1993a. South American Oxybelus II. The emarginatus group (Hymenoptera, Sphecidae). Insecta Mundi, 386: 19-26.

Bohart, R.M. 1993b. South American Oxybelus III. The uniglumis and scutellatus groups (Hymenoptera, Sphecidae). Insecta Mundi, 384: 65-76.

Bohart, R.M. 1993c. A synopsis of Central American and Caribbean Oxybelus (Hymenoptera, Sphecidae). Insecta Mundi, 7: 159-168.

Bohart, R.M. 2000. A review of Gorytini in the Neotropical Region (Hymenoptera: Sphecidae: Bembicinae). Contributions on Entomology International, 4(2): 113-259.

Brandão, C.R.F. 1991. Adendos ao catálogo abreviado das formigas da região Neotropical (Hymenoptera: Formicidae). Revista Brasileira de Entomologia, 35: 319-412.

Brandão, C.R.F.; Esteves, F.A. \& Prado, L.P. 2010. A Catalogue of the Pseudomyrmecinae ants type specimens (Hymenoptera, Formicidae) deposited in the Museu de Zoologia da Universidade de São Paulo, Brazil. Papéis Avulsos de Zoologia, 50: 693-699. D01

Esteves, F.A.; Brandão, C.R.F. \& Prado, L.P. 2011. The type specimens of "Dorylomorph" ants (Hymenoptera, Formicidae: Aenictinae, Ecitoninae, Cerapachyinae, Leptanilloidinae) deposited in the Museu de Zoologia da Universidade de São Paulo, Brazil. Papéis Avulsos de Zoologia, 51: 341357. D0I

Fox, W.J. 1899. Contributions to a knowledge of the Hymenoptera of Brazil. Proceedings of the Academy of Natural Sciences of Philadelphia, 51: 195200, 1900.

Hymenoptera Online (HOL). 2018. Available at: http://hol.osu.edu. Access in: 01/03/2018.

Integrated Taxonomic Information System (ITIS). 2018. Available at: http:// www.itis.gov. Access in: 01/06/2018.

International Commission on Zoological Nomenclature (ICZN). 1999. International Code of Zoological Nomenclature. $4^{\text {a }}$ ed. London, ICZN. Available at: www.nhm.ac.uk/hosted-sites/iczn/code. Access in: 01/06/2018.

Klingenberg, C. \& Brandão, C.R.F. 2005. The type specimens of fungus growing ants, Attini (Hymenoptera, Formicidae, Myrmicinae) deposited in the Museu de Zoologia da Universidade de São Paulo, Brazil. Papéis Avulsos de Zoologia, 45: 41-50. D01

Leclercq, J. 2000. Hyménopteres Sphecides Crabronien d'Amérique Latine du genre Podagritus Spinola, 1851. Notes Fauniques de Gembloux, 37: 3-35.
Melo, G.A.R. 1992. Duas novas espécies de Microstigmus Ducke, 1907 (Hymenoptera, Sphecidae). Revista Brasileira de Entomologia, 36: 663-670.

Melo, G.A.R. \& Evans, H.E. 1993. Two new Microstigmus species (Hymenoptera, Sphecidae), with the description of their parasite, Goniozus microstigmi sp. n. (Hymenoptera, Bethylidae). Proceedings of the Entomological Society of Washington, 95: 258-263.

Melo, G.A.R. \& Matthews, R.W. 1997. Six new species of Microstigmus wasps (Hymenoptera: Sphecidae), with notes on their biology. Journal of Natural History, 31(3): 421-437.

Menke, A.S. \& Richards, O.W. 1983. Trypoxylon latro, new species. The PanPacific Entomologist, 59: 152-162.

Menke, A.S. \& Vardy, C. 1980. A synopsis of the tribe Scapheutini (Hymenoptera: Sphecidae). Papéis Avulsos de Zoologia, 34: 73-83.

Oliveira, M.L.; Fernandez, D.R.R.; Alvarenga, T.M.; Andena, S.R.; Araujo, R.0.; Azevedo, C.0.; Barbosa, D.N.; Bartholomay, P.R.; Costa, V.A.; DalMolin, A.; Fernandes, I.0.; Gadelha, S.S.; Galhardo, F.; Hermes, M.G.; Justino, C.E.L.; Kawada, R.; Kohler, A.; Lara, R.I.R.; Lucena, D.A.A.; Luz, D.R.; Macedo, A., Maragaría, C.;Oliveira, B.G.;Pádua, D.G.;Perioto, N.W.;Pikart, T.G.;Querino, R.B.; Rosa, B.B.; Santos, B.F.; Santos, E.F.; Schoeninger, K.; Smith, D.R.; Somavilla, A.; Tavares, M.T.; Zanella, F.C.V. \& Zilch, K. 2018. Hymenoptera in Taxonomic Catalog of the Brazilian Fauna. PNUD. Available at: http:// fauna.jbrj.gov.br/fauna/faunadobrasil/96. Access in: 01/03/2018.

Onody, H.C.; Kawada, R. \& Brandão, C.R.F. 2014. Type specimens of Hymenoptera deposited in the Museu de Zoologia da Universidade de São Paulo, Brazil (excluding Aculeata). Papéis Avulsos de Zoologia, 54: 391-402. DOI

Prado, L.P. \& Brandão, C.R.F. 2013. A catalogue of Cephalotini ant type specimens (Hymenoptera: Formicidae: Mymicinae) deposited in the Museu de Zoologia da Universidade de São Paulo, Brazil. Papéis Avulsos de Zoologia, 53: 285-293. DOI

Pulawski, W.J. 1974. A revision of the Neotropical Tachysphex Kohl (Hymenoptera, Sphecidae) - Neotropika Inegatunki Rodzaju Tachysphex (Hymenoptera, Sphecidae). Polskie Pismo Entomologiczne, 44: 3-102.

Pulawski, W.J. 2018. Catalog of Sphecidae. California Academy of Sciences. Available at: www.calacademy.org/scientists/projects/catalog-ofsphecidae. Access in: 4/06/2018.

Ramos, K.S.; Kawada, R. \& Brandão, C.R.F. 2015. Type specimens of bees (Hymenoptera, Apidae) deposited in the Museu de Zoologia da Universidade de São Paulo, Brazil. Papéis Avulsos de Zoologia, 55: 335361. DOI

Santos, A.D.;Onody, H.C.;Brandão, C.R.F. 2017. Type specimens of Chrysidoidea (Hymenoptera) deposited in the Museu de Zoologia da Universidade de São Paulo, Brazil. Papéis Avulsos de Zoologia, 57(26): 327-345. D01

Schrottky, C. 1913. Neue südamerikanische Hymenopteren. Deutsch Entomologische Zeitschrift, (1913): 702-708.

Ulysséa, M.A. \& Brandão, C.R.F. 2013. Catalogue of Dacetini and Solenopsidini ant type specimens (Hymenoptera, Formicidae, Myrmicinae) deposited in the Museu de Zoologia da Universidade de São Paulo, Brazil. Papéis Avulsos de Zoologia, 53(14): 187-209. DOI

Ulysséa, M.A.; Prado, L.P. \& Brandão, C.R.F. 2015. Type specimens of the traditional Myrmicinae (Hymenoptera: Formicidae) ant tribes deposited in the Museu de Zoologia da Universidade de São Paulo, Brazil: Adelomyrmecini, Basicerotini, Blepharidattini, Crematogastrini, Formicoxenini, Lenomyrmecini, Myrmicini, Phalacromyrmecini, Pheidolini, Stegomyrmecini, Stenammini and Tetramoriini. Papéis Avulsos de Zoologia, 55(12): 175-204. 